\title{
Potencial alelopático de Ziziphus joazeiro Mart. (Rhamnaceae) sobre a germinação e desenvolvimento de plantas daninhas
}

\author{
Allelopathic potential of Ziziphus joazeiro Mart. (Rhamnaceae) about the germination and \\ development of weeds
}

Potencial alelopático de Ziziphus joazeiro Mart. (Rhamnaceae) sobre la germinación y el desarrollo de malas hierbas

Maria Daniele Pereira Rodrigues ORCID: https://orcid.org/0000-0002-9483-2195 Universidade Regional do Cariri, Brasil E-mail: daniele.hsm@ hotmail.com Cicero dos Santos Leandro ORCID: https://orcid.org/0000-0002-6311-1715 Universidade Regional do Cariri, Brasil E-mail: cicero.leandro2@gmail.com

Ana Karolina Fernandes Silva ORCID: https://orcid.org/0000-0002-8721-5922 Universidade Regional do Cariri, Brasil E-mail: ana.karolina.fer@gmail.com

José Weverton Almeida-Bezerra ORCID: https://orcid.org/0000-0002-0966-9750 Universidade Regional do Cariri, Brasil E-mail: weverton.almeida@urca.br

Danúbio Lopes da Silva

ORCID: https://orcid.org/0000-0002-3168-7437 Universidade Regional do Cariri, Brasil

E-mail: danubio_ls@hotmail.com

Mikael Amaro de Souza

ORCID: https://orcid.org/0000-0001-7085-5870 Universidade Regional do Cariri, Brasil E-mail: mikael.amaro.08@hotmail.com

Maria Aparecida Barbosa Ferreira Gonçalo ORCID: https://orcid.org/0000-0003-4974-2606 Universidade Regional do Cariri, Brasil

E-mail: cida_barba@hotmail.com Luciano Temoteo dos Santos ORCID: https://orcid.org/0000-0002-9215-6832 Universidade Federal do Cariri, Brasil

E-mail: luciano.temoteosantos@gmail.com

Jamile Maria Pereira Bastos Lira de Vasconcelos ORCID: https://orcid.org/0000-0002-0898-0643 Secretaria de Educação e Esportes de Pernambuco, Brasil E-mail: jamilebastoslira@gmail.com Nara Juliana Santos Araújo ORCID: https://orcid.org/0000-0003-0775-4369 Universidade Federal do Cariri, Brasil E-mail: narajuliana6@gmail.com

Geralda de Araújo Lima ORCID: https://orcid.org/0000-0002-6096-0834 Escola de Saúde Pública do Estado do Ceará, Brasil E-mail: geraldabezerra@gmail.com

Viviane Bezerra da Silva

ORCID: https://orcid.org/0000-0003-0581-2609 Universidade Regional do Cariri, Brasil E-mail: viviane.silva@urca.br Maria Arlene Pessoa da Silva ORCID: https://orcid.org/0000-0002-2643-2106 Universidade Regional do Cariri, Brasil E-mail: arlene.pessoa@urca.br 


\begin{abstract}
Resumo
A alelopatia pode ser utilizada no combate às plantas daninhas, as quais podem interferir negativamente nos diversos ambientes naturais e cultivados. Entre as plantas daninhas existentes na Caatinga estão Calotropis procera e Cenchrus echinatus. Desta forma, objetivou-se, avaliar o efeito alelopático de extratos de folhas, caules e raízes de Ziziphus joazeiro na germinação e desenvolvimento das referidas espécies. Foram utilizadas duas porções de $50 \mathrm{~g}$ de cada parte da planta para $500 \mathrm{~mL}$ de $\mathrm{H}_{2} 0$ destilada. O experimento constou de seis tratamentos: extrato de folhas, caules e raízes a $100{ }^{\circ} \mathrm{C}$ (infusão) e a $25^{\circ} \mathrm{C}$ (a frio), seguidos de um grupo controle (água destilada). Os parâmetros analisados foram: Porcentagem de germinação, Índice de Velocidade de Germinação e comprimento do caulículo e radícula. De modo geral, a porcentagem de germinação e o IVG das de $C$. echinatus foram afetados negativamente por todos os tratamentos testados. Com relação ao desenvolvimento de suas plântulas, houve uma interferência significativa no desenvolvimento das radículas. Em relação às sementes de $C$. procera, quando comparadas ao grupo controle, não houve nenhuma diferença estatística nem para a porcentagem de germinação nem para o IVG. Com relação ao desenvolvimento não houve interferência nem para o caulículo nem para a radícula, com exceção do Extrato por infusão a quente que inibiu o desenvolvimento do caulículo. Os resultados mostram que houve interferência alelopática mais eficiente sobre $C$. echinatus principalmente quando submetida ao extrato por infusão da casca da espécie doadora.
\end{abstract}

Palavras-chave: Calotropis procera; Cenchrus echinatus; Fitotoxicidade; Juazeiro; Rhamnaceae.

\begin{abstract}
Allelopathy can be used to combat weeds, which can negatively interfere in various natural and cultivated environments. Among the weeds found in the Caatinga are Calotropis procera and Cenchrus echinatus. Thus, the objective was to evaluate the allelopathic effect of extracts from leaves, stems and roots of Ziziphus joazeiro on the germination and development of these species. Two portions of $50 \mathrm{~g}$ of each part of the plant were used for $500 \mathrm{~mL}$ of distilled H20. The experiment consisted of six treatments: extract of leaves, stems and roots at $100{ }^{\circ} \mathrm{C}$ (infusion) and at $25{ }^{\circ} \mathrm{C}$ (cold), followed by a control group (distilled water). The analyzed parameters were: germination percentage, germination speed index and stem and radicle length. In general, germination percentage and IVG of C. echinatus were negatively affected by all tested treatments. Regarding the development of its seedlings, there was a significant interference in the development of the radicles. Regarding $C$. procera seeds, when compared to the control group, there was no statistical difference neither for germination percentage nor for IVG. Regarding the development, there was no interference neither for the stem nor for the radicle, with the exception of the hot infusion extract which inhibited the stem development. The results show that there was more efficient allelopathic interference on $C$. echinatus especially when subjected to the extract by infusion of the donor species bark.
\end{abstract}

Keywords: Calotropis procera; Cenchrus echinatus; Phytotoxicity; Juazeiro; Rhamnaceae.

\title{
Resumen
}

La alelopatía se puede utilizar para combatir las malas hierbas, que pueden interferir negativamente en diversos entornos naturales y cultivados. Entre las malas hierbas que se encuentran en la Caatinga se encuentran Calotropis procera y Cenchrus echinatus. Así, el objetivo fue evaluar el efecto alelopático de extractos de hojas, tallos y raíces de Ziziphus joazeiro sobre la germinación y desarrollo de estas especies. Se utilizaron dos porciones de $50 \mathrm{~g}$ de cada parte de la planta para $500 \mathrm{~mL}$ de $\mathrm{H} 2 \mathrm{O}$ destilada. El experimento consistió en seis tratamientos: extracto de hojas, tallos y raíces a $100{ }^{\circ} \mathrm{C}$ (infusión) y a $25^{\circ} \mathrm{C}$ (frío), seguido de un grupo control (agua destilada). Los parámetros analizados fueron: porcentaje de germinación, índice de velocidad de germinación y longitud de tallo y radícula. En general, el porcentaje de germinación y la IVG de $C$. echinatus se vieron afectados negativamente por todos los tratamientos probados. En cuanto al desarrollo de sus plántulas, hubo una interferencia significativa en el desarrollo de las radículas. Con respecto a las semillas de C. procera, en comparación con el grupo control, no hubo diferencia estadística ni para el porcentaje de germinación ni para el IVG. En cuanto al desarrollo, no hubo interferencia ni para el tallo ni para la radícula, a excepción del extracto de infusión caliente que inhibió el desarrollo del tallo. Los resultados muestran que hubo una interferencia alelopática más eficiente sobre $C$. echinatus especialmente cuando se sometió al extracto por infusión de la corteza de la especie donante.

Palabras clave: Calotropis procera; Cenchrus echinatus; Fitotoxicidad; Juazeiro; Rhamnaceae.

\section{Introdução}

A alelopatia é definida como a capacidade de interação entre duas ou mais plantas, onde uma pode interferir negativamente ou positivamente no desenvolvimento e germinação de outra (Hierro et al., 2021). Essa interação é resultado da ação de biomoléculas denominadas aleloquímicos, que são produzidos pela planta e liberados no ambiente (Silva et al., 2021).

Esses aleloquímicos são compostos secundários e podem agir de forma direta ou indireta, sendo encontrados em concentrações distintas nos diferentes tecidos da planta (Li et al., 2010; Ferguson et al., 2013). Esses aleloquímicos, segundo 
Rice (1984) são liberados por volatilização, lixiviação, exsudação radicular e/ou decomposição de resíduos vegetais, e podem afetar diversos processos fisiológicos, tais como crescimento e desenvolvimento.

A ação dos aleloquímicos em determinadas plantas vem sendo intensamente estudadas nas duas últimas décadas, visto que plantas detentoras de compostos capazes de inibir o crescimento de outras, podem ser utilizadas como herbicidas naturais eficientes (Zhang et al., 2021). Esses bioherbicidas tornam-se uma alternativa de controle e manejo de plantas daninhas (Chen et al., 2017).

Segundo Gaba et al. (2017) as plantas daninhas são um grande problema para a agricultura em virtude de serem responsáveis por parte dos prejuízos nas grandes culturas. Desta forma, estudos relacionados ao uso da alelopatia para a produção de herbicidas naturais que auxiliem no controle e manejo das mesmas são de grande importância. Vários estudos comprovam o potencial alelopático de espécies de Caatinga frente às plantas daninhas (Silva et al., 2018; Leandro et al., 2019; Almeida-Bezerra et al., 2020).

Ziziphus joazeiro Mart. pertencente à família Rhamnaceae Juss., é uma planta característica da Caatinga encontrada principalmente na região Nordeste. É utilizada localmente para a produção de lenha e carvão, arborização de ruas e jardins (Oliveira et al., 2020), e possui grande valor econômico já que é utilizada para a produção de sabão e de xampu e apresenta grandes propriedades medicinais principalmente contra problemas dermatológicos e do sistema respiratório (Andrade et al., 2019; Albuquerque et al., 2007).

Calotropis procera (Aiton) W.T., (Apocynaceae), conhecida popularmente como papai-Noel, é nativa do Sudoeste Asiático e África, sendo comumente encontrada na região Nordeste do Brasil, comportando-se como invasora do meio natural (Costa et al., 2009). Cenchrus echinatus L. (Poaceae) é uma espécie de grande importância na invasão de culturas, sendo conhecida popularmente como carrapicho, é difundida em quase todas as regiões de cultivo do Brasil. Constatou-se infestação desta espécie em diversos campos comerciais, como amendoim, feijão, mandioca, soja cana de açúcar cebola, tomate, bem como em pastagens (Silva et al., 2015). As espécies daninhas supracitadas escolhidas para o estudo além de serem conhecidas por ocorrerem na invasão de culturas, também são encontradas invadindo áreas de Caatinga (Fabricante, 2013; Fabricante et al 2016). Tais espécies são controladas nestes ambientes através de herbicidas sintéticos, sendo estes produtos conhecidos por causarem danos ambientais e à saúde humana (Leandro et al., 2019).

Considerando a escassez de trabalhos relacionados ao potencial alelopático de Z. joazeiro para o controle de plantas daninhas e levando em conta a importância de tais estudos, esse trabalho tem como objetivo avaliar o potencial alelopático dos extratos das folhas, cascas e raizes de Z. joazeiro sobre a germinação e desenvolvimento das plantas daninhas $C$. procera e $C$. echinatus, além de fazer um levantamento dos compostos secundários já isolados da referida espécie doadora.

\section{Metodologia}

\subsection{Coleta e identificação do material botânico}

O material botânico de Ziziphus joazeiro (folhas, caule e raiz para produção do extrato e ramos floríferos para identificação) foi coletado na cidade do Crato-CE em setembro de 2016 (S 07¹4.300’ e W 039 24.969'). Todo material coletado foi acondicionado em sacos plástico de $50 \mathrm{~L}$ devidamente lacrados e etiquetados como forma de facilitar o transporte da amostra para posterior prensagem em local mais apropriado. Os ramos com flores e frutos foram devidamente herborizados conforme a metodologia de Rezende et al. (2017), e após identificação a partir de comparações com materiais pré-existentes na coleção, foram incorporados ao acervo do Herbário Caririense Dárdano de Andrade-Lima - HCDAL da Universidade Regional do Cariri - URCA sob número de registro 13.783. 


\subsection{Preparação dos extratos}

Os testes alelopáticos foram conduzidos no Laboratório de Botânica Aplicada-LBA do Departamento de Ciências Biológicas da Universidade Regional do Cariri-URCA. Para a preparação dos extratos foram utilizados tecidos vegetativos de folhas, cascas e raízes de $Z$. joazeiro.

Para a preparação dos extratos foram utilizadas duas porções de $50 \mathrm{~g}$ de cada órgão da planta os quais foram acondicionadas separadamente em seis beckeres. Cada parte da planta recebeu um tratamento a quente $(500 \mathrm{~mL}$ de água destilada aquecida à $\left.100{ }^{\circ} \mathrm{C}\right) \mathrm{e}$ um a frio $\left(500 \mathrm{~mL}\right.$ de água destilada à temperatura ambiente $\left.25^{\circ} \mathrm{C}\right)$, totalizando seis extratos (tratamentos) e um grupo controle o qual constou somente de água destilada. Sendo os tratamentos: (1) Extrato por infusão a Quente da Casca (EQC); (2) Extrato a Frio da Casca (EFC); (3) Extrato por infusão a Quente das Folhas (EQF); (4) Extrato a Frio das Folhas (EFF); (5) Extrato por infusão a Quente da Raiz (EQR); (6). Extrato Frio da Raiz (EFR) (Leandro et al., 2019).

O período de infusão das partes vegetais foi de $30 \mathrm{~min}$. Decorrido esse tempo, cada extrato foi triturado em liquidificador industrial por $1 \mathrm{~min}$, sendo em seguida filtrado com auxílio de funil e algodão e posteriormente centrifugado para a retirada de todo o material fibroso. Foram aferidos o pH e o potencial osmótico de cada extrato (Leandro et al., 2019).

\subsection{Características físico-químicas}

O potencial hidrogeniônico dos extratos das folhas, cascas do caule e raízes foram analisadas com o auxílio do pHmetro. A medição da concentração molar dos referidos extratos foi realizada por meio do método de Chardakov (Villela et al., 1991). Os dados referentes a osmolaridade foram obtidas em mOsm/kg e convertidos para pressão osmótica (MPa) (Larcher, 2004).

\subsection{Testes alelopáticos}

Foram utilizadas como espécies receptoras $C$. procera e C. echinatus, sendo estas, espécies daninhas coletadas em terrenos baldios próximos à Universidade Regional do Cariri.

Os bioensaios foram realizados em placas de Petri, forradas com papel germitest umedecidos com $3 \mathrm{~mL}$ de extrato (tratamentos) ou $3 \mathrm{~mL}$ de água destilada (grupo controle). Cada tratamento constou de quatro repetições de 25 sementes cada, de acordo com as instruções de regras para análises de semente do Ministério da Agricultura Pecuária e Abastecimento (Brasil, 2009). O experimento foi realizado em câmara de germinação do tipo B.O.D (biochemical oxygen demand) com fotoperíodo de 12 hrs e teve duração de sete dias para C. procera e cinco para C. echinatus. A contagem de sementes germinadas foi realizada a cada 24 hrs (Leandro et al., 2019).

\subsection{Variáveis analisadas}

Foram analisados a porcentagem de germinação, Índice de Velocidade de Germinação-IVG, comprimento do caulículo e comprimento da radícula, conforme Popinigis (1985).

\subsubsection{Porcentagem de germinação}

A porcentagem de germinação (PG) foi calculada utilizando a fórmula proposta por Azevedo Neto (2010): G=(N/A) * 100.

Onde: $\mathbf{N}$ - Refere-se ao número total de sementes germinadas ao final do experimento

A - Refere-se ao número total de sementes semeadas. 


\subsection{2 Índice de Velocidade de Germinação-IVG}

O número de sementes germinadas foi contado a cada $24 \mathrm{hrs}$ durante sete dias para $C$. procera e cinco dias para $C$. echinatus, sendo calculado de acordo Maguire (1962). A partir desse registro foi obtido o IVG que foi determinado através da seguinte fórmula:

$$
I V G=\sum_{i=1}^{n}\left(\frac{n i}{i}\right)
$$

Onde: $\mathbf{n i}=$ número de sementes germinadas no dia i.

$\mathbf{i}=$ número de dias

\subsubsection{Comprimento do caulículo e da radícula}

Após o período do teste, foram coletadas aleatoriamente cinco plântulas de cada repetição de $C$. procera e $C$. echinatus as quais foram submetidas a medição do comprimento do caulículo e da radícula com auxílio de um paquímetro digital. Os resultados foram expressos em milímetros (mm) (Leandro et al., 2019).

\subsection{Análise estatística}

Para a análise estatística dos dados foi utilizado o programa GraphPad Prism versão 6.0, com análise de variância (ANOVA) de uma via e comparação das médias através de Análise de Regressão ou pelo Teste de Tukey a 5\% de probabilidade.

\section{Resultados e Discussão}

\subsection{Parâmetros físico-químicos}

Todos os extratos apresentaram pH ácido, sendo ajustados para um pH entre 6,0 e 7,5 já que de acordo com Macias et al. (2000), esse é o padrão aceitável para o desenvolvimento e germinação de plântulas em testes de alelopatia (Tabela 1).

Com relação à osmolaridade, todos os extratos apresentaram pouco soluto em sua constituição. Vale ressaltar que níveis elevados de osmolaridade podem inibir ou retardar o crescimento e desenvolvimento de plântulas (Ortiz et al., 2014) desse modo, as atividades alelopáticas negativas observadas não estão relacionadas ao nível de soluto, já que todos os valores se aproximaram de zero (Tabela 1).

Tabela 1 - Valores fisíco-químicos dos extratos, a quente e a frio de diferentes partes de Z. joazeiro usados nos bioensaios de germinação de C. procera e C. echinatus.

\begin{tabular}{cccc}
\hline Tratamento & $\mathbf{p H}$ & $\mathbf{p H}$ ajustado & Osmolaridade Mpa \\
\hline EQC & 5,74 & 6,67 & $-0,018$ \\
EFC & 5,79 & 6,82 & $-0,061$ \\
EQF & 6,14 & 6,51 & $-0,057$ \\
EFF & 6,11 & 6,75 & $-0,034$ \\
EQR & 5,61 & 6,81 & $-0,025$ \\
EFR & 5,52 & 6,61 & $-0,018$ \\
\hline
\end{tabular}

Legenda: EQC: Extrato por infusão a Quente das Cascas; EFC: Extrato por infusão a Frio das Cascas; EQF: Extrato por infusão a Quente das Folhas; EFF: Extrato por infusão a Frio das Folhas; EQR: Extrato por infusão a Quente das Raízes. EFR: Extrato por infusão a Frio das Raízes. Fonte: Autores. 


\subsection{Porcentagem de germinação}

Sobre a porcentagem de germinação, nas sementes de C. procera não houve interferência significativa, apesar dos valores variarem entre $72,8 \%$ e $97,5 \%$ (Figura 1). Já para as sementes de C. echinatus todos os extratos interferiram significativamente na porcentagem de germinação, sendo que os extratos por infusão a quente da casca, frio da casa e por infusão a quente das folhas tiveram os melhores resultados quando comparado aos demais (Figura 2).

Figura 1 - Porcentagem de germinação das sementes de C. procera submetidas aos extratos de Z. joazeiro.

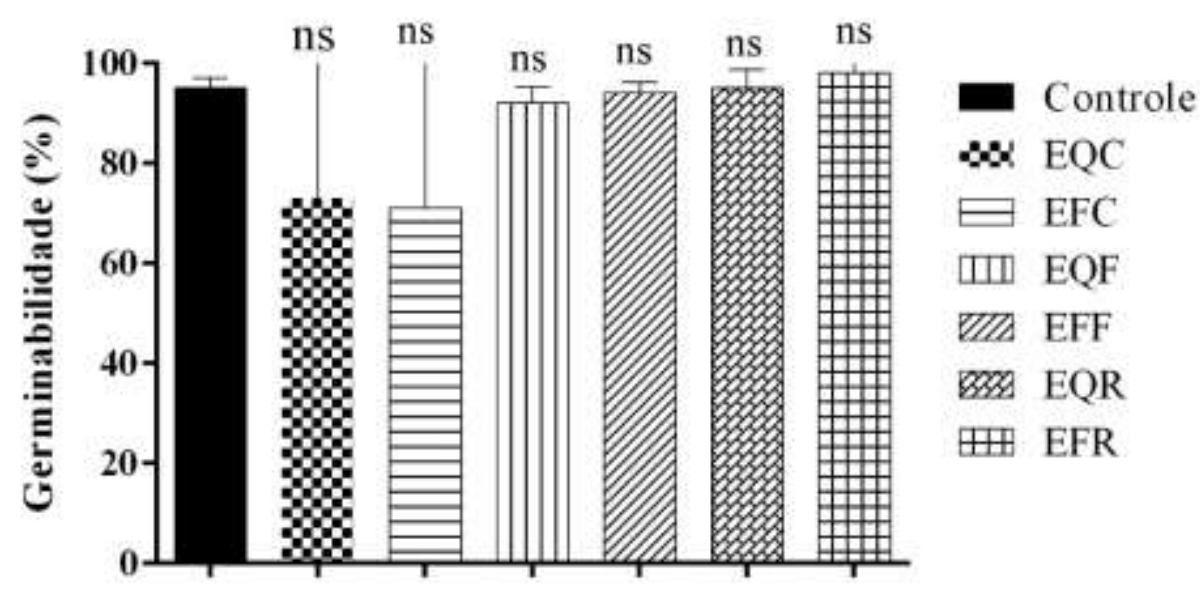

Legenda: EQC: Extrato por infusão a Quente das Cascas; EFC: Extrato por infusão a Frio das Cascas; EQF: Extrato por infusão a Quente das Folhas; EFF: Extrato por infusão a Frio das Folhas; EQR: Extrato por infusão a Quente das Raízes. EFR: Extrato por infusão a Frio das Raízes. Análise de variância (ANOVA) One-way. Média ( \pm desvio padrão). ns: sem significância estatística. * $p<0,05$ em comparação com o controle. Fonte: Autores.

Figura 2 - Porcentagem de germinação das sementes de C. echinatus submetidas aos extratos de Z. joazeiro

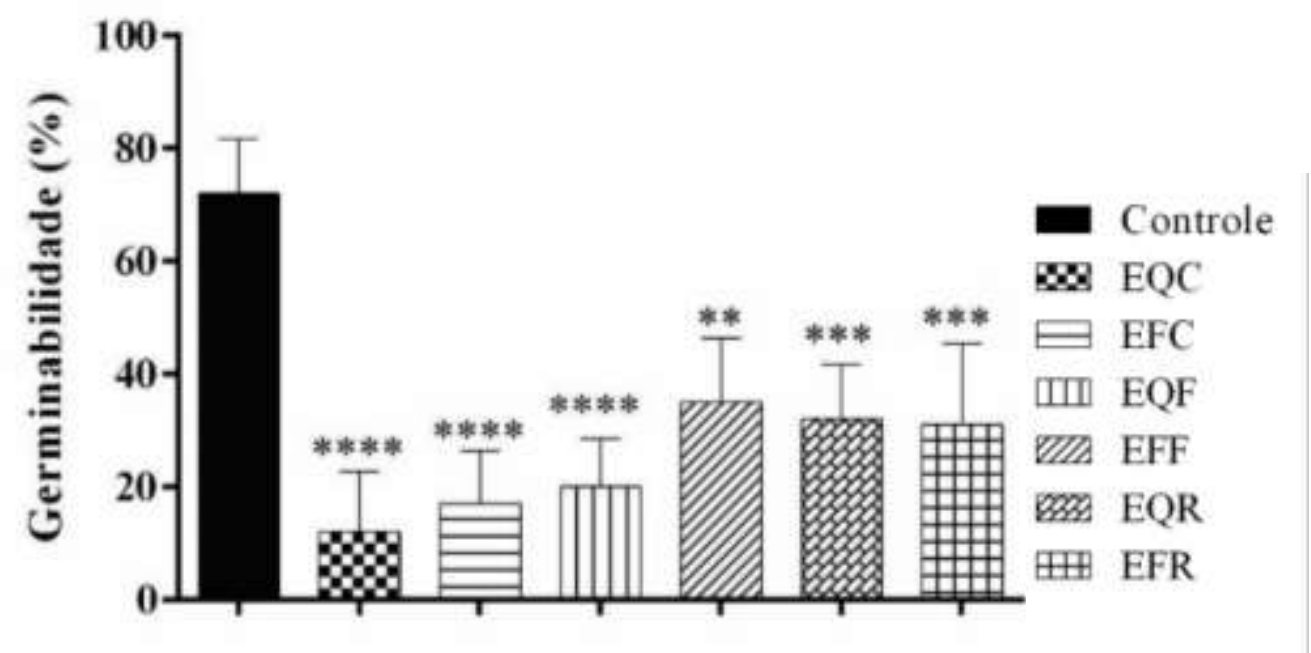

Legenda: EQC: Extrato por infusão a Quente das Cascas; EFC: Extrato por infusão a Frio das Cascas; EQF: Extrato por infusão a Quente das Folhas; EFF: Extrato por infusão a Frio das Folhas; EQR: Extrato por infusão a Quente das Raízes. EFR: Extrato por infusão a Frio das Raízes. Análise de variância (ANOVA) One-way. Média ( \pm desvio padrão). ns: sem significância estatística. * $p<0,05$ em comparação com o controle. Fonte: Autores.

Ao testar o efeito dos extratos aquosos de Z. joazeiro sobre a germinação de sementes de Lycopersicon esculentum Mill., Parente et al. (2015) mostraram que os extratos não afetaram a porcentagem de germinação das sementes. Já Araújo 
(2016), ao analisar o efeito alelopático das folhas e cascas de Z. joazeiro sobre a germinação de Lactuca sativa L. constatou que os extratos afetaram negativamente a porcentagem de germinação. Esses resultados mostram que os extratos de Z. joazeiro podem se comportar de modo diferente dependo da espécie sobre a qual forem testados, o que fica evidente quando o mesmo extrato interferiu na porcentagem de germinação das sementes de C. echinatus e obteve resultado contrário em relação as sementes de $C$. procera.

\section{3 Índice de Velocidade de Germinação (IVG)}

Para as sementes de $C$. procera não houve diferença estatística no que diz respeito ao índice de velocidade de germinação quando comparado ao grupo controle (Tabela 2). É provável que as sementes de C. procera sejam mais resistentes aos efeitos dos extratos testados, possivelmente por ambas serem do grupo vegetal o das Eudicotiledôneas (APG IV, 2016).

O Índice de velocidade de germinação em C. echinatus (Monocotiledônea) foi afetado negativamente em todos os extratos testados. Verificou-se que as sementes submetidas ao extrato por infusão a quente da casca apresentaram uma menor velocidade de germinação quando comparadas ao grupo controle. (Tabela 2), por serem de grupos vegetais distintos (APG IV, 2016).

Extratos de diferentes órgãos vegetais podem ser mais eficientes em reduzir a velocidade de germinação de sementes em comparação a outro, isso pode ser explicado pela concentração de metabólitos secundários presentes os quais podem ser diferentes em cada órgão da planta (Silva \& Santos, 2012). Fatores como temperatura e luminosidade também podem resultar em retardo na velocidade de germinação (Araújo et al. 2003). Segundo Hoagland \& Williams (2004) esse atraso na velocidade de germinação é um indicador do efeito alelopático sobre o alongamento e divisão celular e que pode ocorrer por meio da ativação de mecanismos de desintoxicação celular.

Tabela 2 - Índice de Velocidade de Germinação- IVG das sementes de C. procera e C. echinatus submetidas aos diferentes extratos de Z. joazeiro.

\begin{tabular}{ccc}
\hline \multirow{2}{*}{ TRATAMENTOS } & \multicolumn{2}{c}{ IVG } \\
\cline { 2 - 3 } & Calotropis procera & Cenchrus echinatus \\
\hline EQC & $3,21 \pm 1,89 \mathrm{a}$ & $0,94 \pm 0,92 \mathrm{~b}$ \\
EQF & $3,53 \pm 2,39 \mathrm{a}$ & $1,90 \pm 1,15 \mathrm{bc}$ \\
EFF & $5,93 \pm 1,02 \mathrm{a}$ & $2,07 \pm 0,99 \mathrm{bc}$ \\
EQR & $6,57 \pm 0,84$ & $3,58 \pm 1,35 \mathrm{bc}$ \\
EFR & $7,37 \pm 0,51 \mathrm{a}$ & $3,70 \pm 1,60 \mathrm{c}$ \\
Controle $\left(\mathbf{H}_{2} \mathbf{O}\right)$ & $6,98 \pm 1,04 \mathrm{a}$ & $3,20 \pm 1,33 \mathrm{bc}$ \\
\hline
\end{tabular}

Legenda: EQC: Extrato por infusão a Quente das Cascas; EFC: Extrato por infusão a Frio das Cascas; EQF: Extrato por infusão a Quente das Folhas; EFF: Extrato por infusão a Frio das Folhas; EQR: Extrato por infusão a Quente das Raízes. EFR: Extrato por infusão a Frio das Raízes. Médias seguidas da mesma letra em coluna não diferem entre si a 5\% de probabilidade, pelo teste de Tukey. Fonte: Autores (2021).

\subsection{Desenvolvimento do caulículo e radícula}

Com relação ao desenvolvimento do caulículo, tanto para $C$. echinatus como para $C$. procera, não houve diferença significativa quando comparado ao grupo controle (Figuras 3 e 4), com exceção ao extrato por infusão a quente da casca que interferiu negativamente no desenvolvimento do caulículo das sementes de C. procera (Figura 4).

A metodologia aplicada para a obtenção dos extratos pode influenciar na forma com que as substâncias aleloquímicas 
são extraídas, talvez o extrato por infusão a quente tenha sido o único a extrair substâncias capazes de interferir do desenvolvimento do caulículo de C. procera. Fatores externos também podem influenciar nos resultados obtidos já que a alelopatia é resultado da ação combinada de vários fatores (Pires \& Oliveira, 2001).

Figura 3 - Desenvolvimento do caulículo de plântulas de C. echinatus submetidas aos extratos de Z. joazeiro.

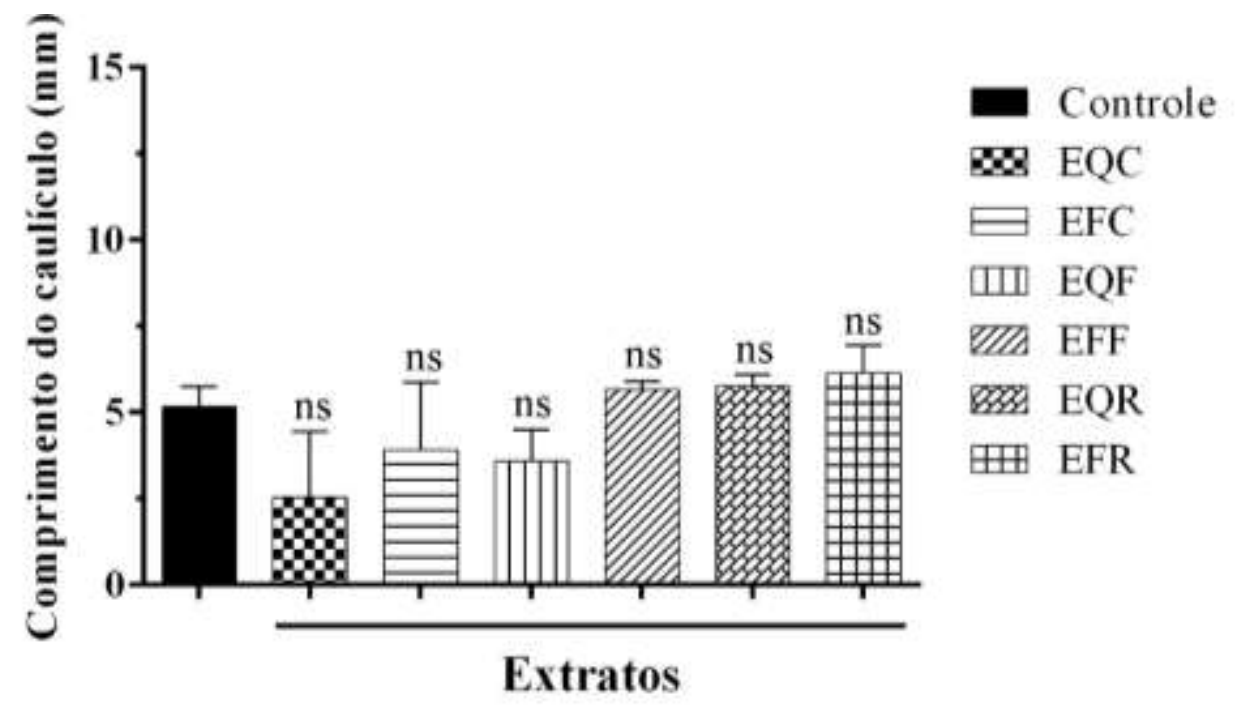

Legenda: EQC: Extrato por infusão a Quente das Cascas; EFC: Extrato por infusão a Frio das Cascas; EQF: Extrato por infusão a Quente das Folhas; EFF: Extrato por infusão a Frio das Folhas; EQR: Extrato por infusão a Quente das Raízes. EFR: Extrato por infusão a Frio das Raízes. Análise de variância (ANOVA) One-way. Média ( \pm desvio padrão). ns: sem significância estatística. * $p<0,05$ em comparação com o controle. Fonte: Autores (2021).

Figura 4 - Desenvolvimento do caulículo de plântulas de Calotropis procera submetidas aos extratos de Ziziphus joazeiro.

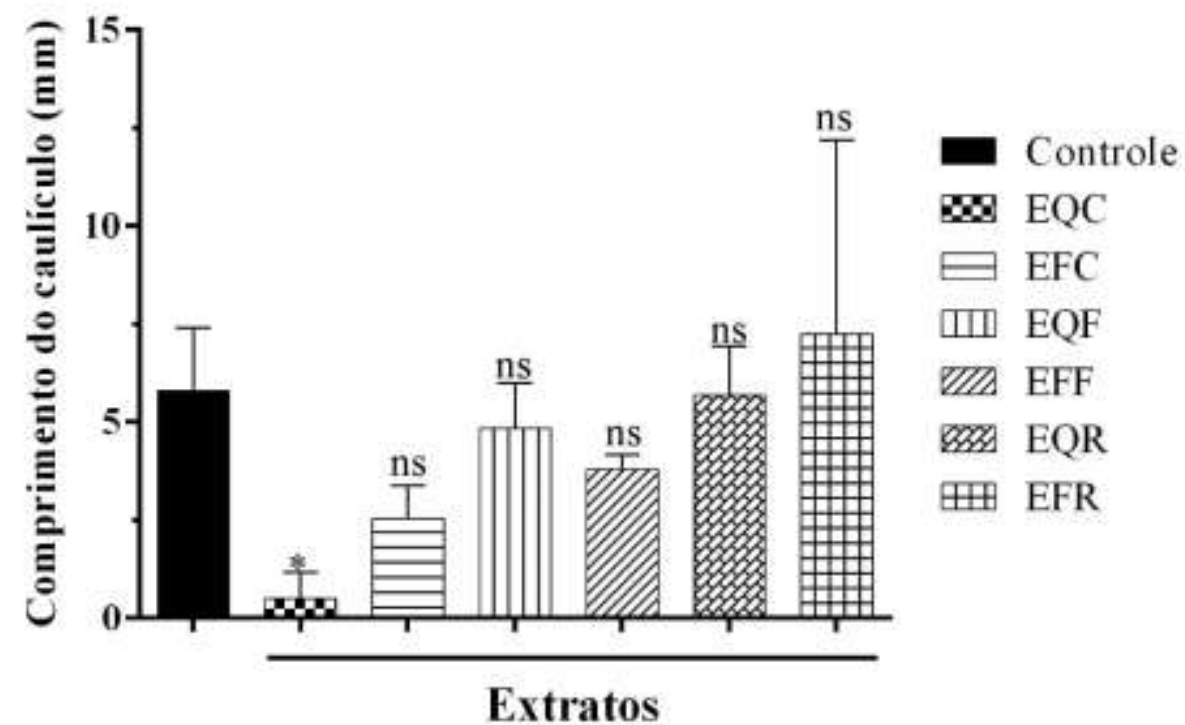

Legenda: EQC: Extrato por infusão a Quente das Cascas; EFC: Extrato por infusão a Frio das Cascas; EQF: Extrato por infusão a Quente das Folhas; EFF: Extrato por infusão a Frio das Folhas; EQR: Extrato por infusão a Quente das Raízes. EFR: Extrato por infusão a Frio das Raízes. Análise de variância (ANOVA) One-way. Média ( \pm desvio padrão). ns: sem significância estatística. *p<0,05 em comparação com o controle. Fonte: Autores (2021). 
Para o desenvolvimento da radícula não houve interferência em C. procera (Figura 5), porém em C. echinatus todos os extratos inibiram significativamente o desenvolvimento da radícula, onde os extratos por infusão a quente e a frio das cascas obtiveram os melhores resultados (Figura 6). Esse resultado corrobora com Oliveira et al. (2012) que testou o extrato bruto extraído a $100{ }^{\circ} \mathrm{C}$ e a $25^{\circ} \mathrm{C}$ das folhas e cascas de Z. joazeiro em sementes de L. sativa mostrando que os extratos das cascas inibiram significativamente o comprimento da radícula das plântulas oriundas das sementes germinadas.

Figura 5 - Desenvolvimento da radícula de plântulas de C. procera submetidas aos extratos de Z. joazeiro.

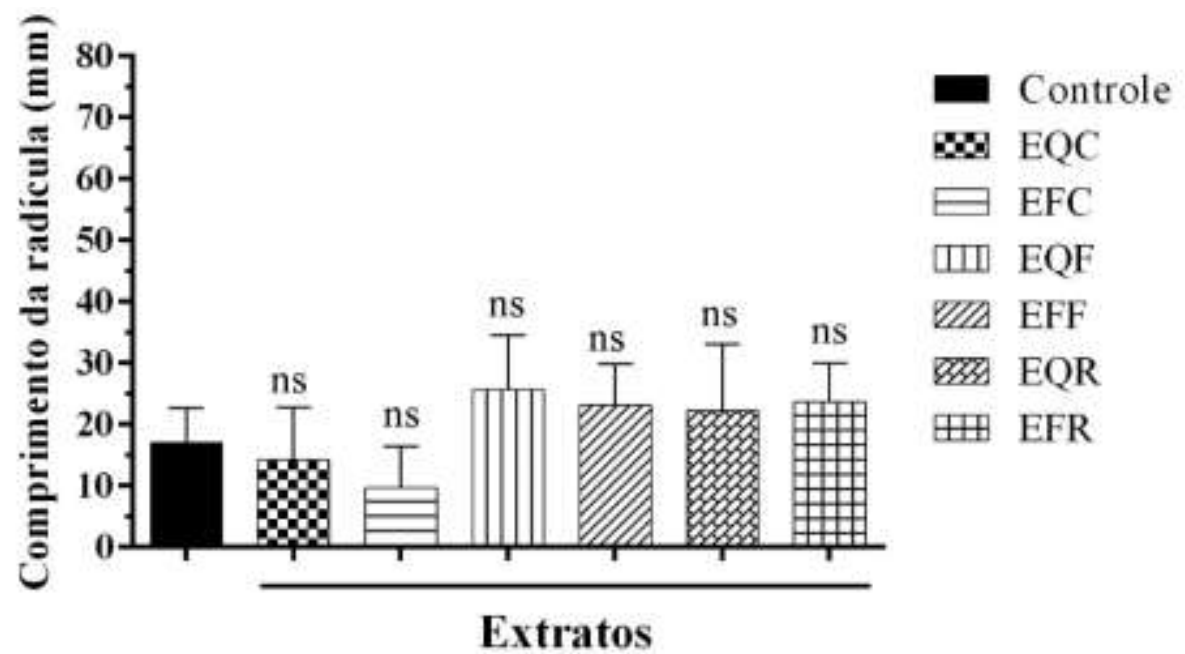

Legenda: EQC: Extrato por infusão a Quente das Cascas; EFC: Extrato por infusão a Frio das Cascas; EQF: Extrato por infusão a Quente das Folhas; EFF: Extrato por infusão a Frio das Folhas; EQR: Extrato por infusão a Quente das Raízes. EFR: Extrato por infusão a Frio das Raízes. Análise de variância (ANOVA) One-way. Média ( \pm desvio padrão). ns: sem significância estatística. *p<0,05 em comparação com o controle. Fonte: Autores (2021).

Figura 6 - Desenvolvimento da radícula de plântulas de C. echinatus submetidas aos extratos de Z. joazeiro.

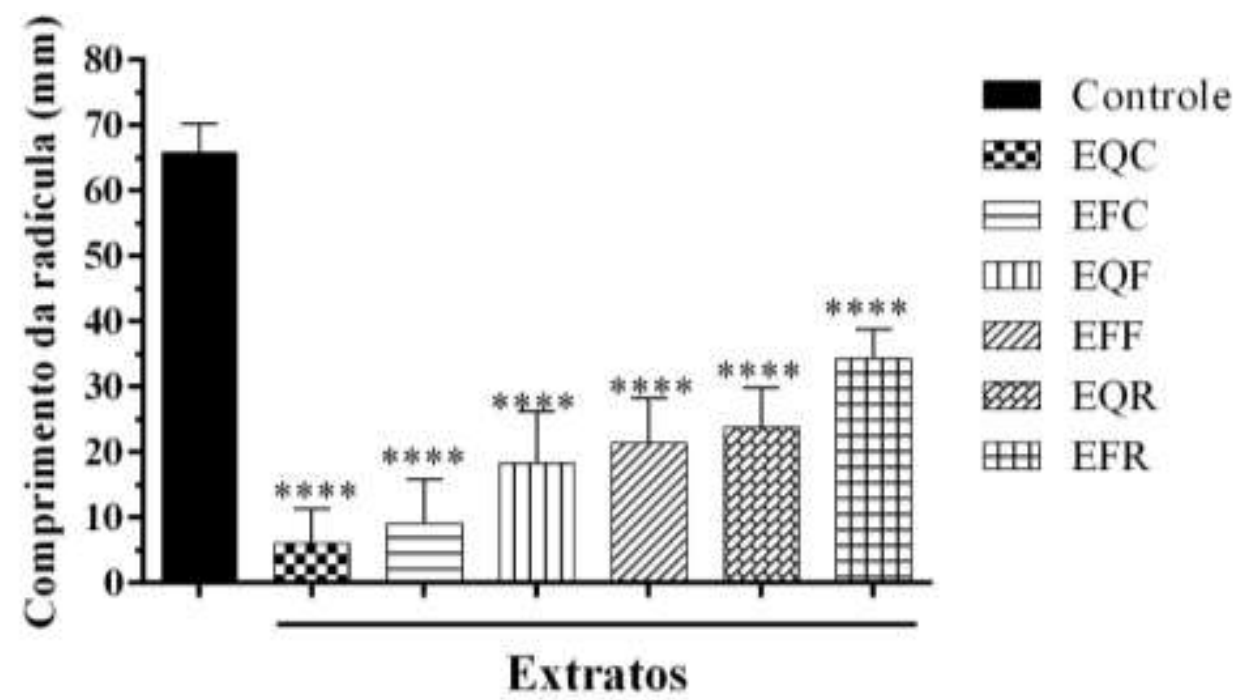

Legenda: EQC: Extrato por infusão a Quente das Cascas; EFC: Extrato por infusão a Frio das Cascas; EQF: Extrato por infusão a Quente das Folhas; EFF: Extrato por infusão a Frio das Folhas; EQR: Extrato por infusão a Quente das Raízes. EFR: Extrato por infusão a Frio das Raízes. Análise de variância (ANOVA) One-way. Média ( \pm desvio padrão). ns: sem significância estatística. * $p<0,05$ em comparação com o controle. Fonte: Autores (2021).

Ferreira e Áquila (2000) asseveram que as raízes são mais sensíveis aos efeitos dos aleloquímicos por estarem em 
contado mais íntimo com o substrato e por serem responsáveis pela absorsão dos compostos bioativos presentes no ambiente. Os aleloquímicos muitas vezes podem bloquear essa absorção de nutrientes levando a redução do crescimento radicular. Esse efeito inibitório no crescimento da radícula é considerado uma das características que melhor indica a fitotoxidade dos extratos vegetais (Turk \& Tawaha, 2002; Santos \& Resende, 2008).

Extratos de diferentes órgãos podem apresentar resultados distintos na germinação e desenvolvimento de diferentes espécies, isso reforça a tese de Prati e Bossdorf (2004) de que a alelopatia é uma relação espécie-específica e que espécies diferentes respondem de formas distintas a presença de um mesmo aleloquímico. Todas as partes das plantas armazenam aleloquímicos porém a quantidade pode variar de acordo com a natureza química, a idade e o órgão vegetal (Almeida et al., 2008), desta forma, extratos obtidos a partir de partes diferentes do mesmo vegetal pode interferir de formas distintas no crescimento e desenvolvimento de determinada planta.

Os estudos sobre os compostos secundários de Z. joazeiro estão mais voltados para as folhas e cascas da referida espécie, tais informações podem ser observadas na Tabela 3. Melo et al. (2012) comprovou, por meio de investigação das classes metabólicas, a presença de alcaloides, saponinas, triterpenos e taninos nas folhas, e saponina e triterpenos nas cascas do caule de Z. joazeiro, compostos estes que de acordo com Ferreira e Áquila (2000) atuam como aleloquímicos.

Tabela 3 - Compostos químicos isolados de Ziziphus joazeiro Mart. (Rhamnaceae Juss)

\begin{tabular}{l|l|l}
\hline \multicolumn{2}{c}{ Órgão vegetal } & \multicolumn{1}{c}{ Compostos encontrados } \\
\hline \multirow{2}{*}{ Folhas } & $\begin{array}{l}\text { Alcalóides, saponinas, triterpenos e } \\
\text { taninos. }\end{array}$ & Melo et al. (2012) \\
\cline { 2 - 3 } & Flavonóides, alcaloides e triterpenos. & Silva (2009) \\
\cline { 2 - 3 } & $\begin{array}{l}\text { Ácidos fenóicos, flavonoides, taninos } \\
\text { e saponinas. }\end{array}$ & Brito (2015) \\
\hline \multirow{2}{*}{ Casca } & Saponina e triterpenos. & Melo et al. (2012) \\
\cline { 2 - 3 } & Flavonóides e triterpenos & Silva (2009) \\
\cline { 2 - 3 } & Saponinas, triterpenos, & Brito (2015) \\
\hline \multirow{2}{*}{ Raíz } & Flavonoides e alcaloides.* & Souza Neto (2016) \\
\hline
\end{tabular}

Legenda: * Compostos encontrados nas raízes de espécies do gênero Ziziphus. Fonte: Autores.

Brito (2015) através de cromatografia líquida de alta eficiência mostrou que as folhas de Z. joazeiro apresentam grande quantidade de flavonóides e que suas cascas são ricas em saponinas e taninos. Os taninos são os compostos com propriedades alelopáticas mais comumente encontrados nos vegetais e estão envolvidos no mecanismo de defesa das plantas (Mendonça, 2008).

Não foram encontrados estudos sobre os compostos químicos presentes nas raízes de Z. joazeiro, porém Souza Neto (2016) afirma que os extratos das raízes das espécies pertencentes ao gênero Ziziphus Mill., frequentemente apresentam flavonóides e alcaloides, grupos de compostos de reconhecida ação alelopática.

A presença dos compostos acima referidos encontrados nos diferentes órgãos de Z. joazeiro mostram que essa espécie tem grande potencial para a alelopatia, já que muitos desses são considerados aleloquímos e podem interferir negativamente ou positivamente no desenvolvimento e na germinação de espécies vizinhas, provavelmente a presença de tais compostos foi um dos fatores responsáveis pelos resultados obtidos nos bioensaios desenvolvidos nessa pesquisa.

\section{Conclusão}

O extrato por infusão a quente da casca de $Z$. joazeiro inibiu o desenvolvimento do caulículo das sementes de $C$. procera. Os extratos por infusão a quente das diferentes partes de Z. joazeiro proporcionaram maiores efeitos inibitórios sobre 
a germinação e desenvolvimento de C. echinatus em relação aos extratos por infusão a frio.

Provavelmente esses tratamentos possibilitaram a extração de uma maior quantidade de aleloquímicos responsáveis por tais efeitos. Possivelmente, as sementes de C. echinatus são mais sensíveis a ação dos metabólitos presentes na espécie testada por ser do grupo vegetal das Monocotiledôneas, já que os resultados para $C$. procera não foram tão significativos quanto para C. echinatus, provavelmente por ambas pertencerem ao grupo das Eudicotiledôneas.

Sendo assim, os extratos de $Z$. joazeiro tem grande potencial para ser utilizado no combate a planta daninha $C$. echinatus e possivelmente de outras Monocotiledôneas. No entanto, são necessárias pesquisas mais aprofundadas, a fim de identificar as substâncias específicas responsáveis pela ação detectada nos bioensaios, para uma possível utilização das mesmas na fabricação de bioherbicidas.

\section{Referências}

Albuquerque, U. P., De Medeiros, P. M., De Almeida, A. L. S., Monteiro, J. M., Neto, E. M. D. F. L., de Melo, J. G., \& Dos Santos, J. P. (2007). Medicinal plants of the caatinga (semi-arid) vegetation of NE Brazil: a quantitative approach. Journal of ethnopharmacology, 114(3), 325-354.

Almeida, G. D., Zucoloto, M., Caldas Zetun, M., Coelho, I., \& Moreira Sobreir, F. (2008). Estresse oxidativo em células vegetais mediante aleloquímicos. Revista Facultad Nacional de Agronomía Medellín, 61(1), 4237-4247.

Almeida-Bezerra, J. W., Oliveira, F. A. M., Nascimento, G. M. S., Pereira, K. S., Leandro, C. S., Correia, D. B; \& Silva, M. A. P. (2020). Allelopathy of Mesosphaerum suaveolens (bamburral) front to seeds of Pilosocereus gounellei subsp. Gounellei (xique-xique). Revista Cubana de Plantas Medicinales, 25(s/n), e863-e863.

Andrade, J. C., Silva, A. R. P., Santos, A. T. L., Freitas, M. A., Carneiro, J. N. P., Gonçalo, M. I. P., \& Coutinho, H. D. M. (2019). UPLC-MS-ESI-QTOF characterization and evaluation of the antibacterial and modulatory antibiotic activity of Ziziphus joazeiro Mart. aqueous extracts. South African Journal of Botany, 123(s/n), 105-112.

APG The Angiosperm Phylogeny Group (2016) An update of the Angiosperm Phylogeny Group classification for the orders and families of flowering plants: APG IV. Botanical Journal of the Linnean Society, 181: 1-20.

Araújo Neto, J. C., Aguiar, I. B., \& Ferreira, V. M. (2003). Efeito da temperatura e da luz na germinação de sementes de Acacia polyphylla DC. Brazilian Journal of Botany, 26(s/n), 249-256.

Araújo, M. N. S. (2016). Alelopatia do extrato de folhas e cascas de juazeiros (Ziziphus joazeiro Mart. - Rhamnaceae) proveniente da localidade do Riacho da légua-Ba. (Trabalho de Conclusão de Curso). Faculdade Maria Nilza, Salgueiro, PE, Brasil.

Azevedo Neto, E. N. (2010). Potencial alelopático de leucena e de sabiá na germinação, na emergência e no crescimento inicial do sorgo. (Monografia Graduação em Engenharia Florestal). Universidade Federal de Campina Grande, Patos, PB, Brasil.

Brasil (2009). Regras para análise de sementes. Ministério da Agricultura Pecuária e Abastecimento. Secretaria de Defesa Agropecuária. 398p.

Brito, I. C. A., \& Santos, D. R. (2012). Alelopatia de espécies arbóreas da caatinga na germinação e vigor de sementes de feijão macacar. Revista Verde de Agroecologia e Desenvolvimento Sustentável, 7(1), 129-140.

Brito, S. M., Coutinho, H. D., Talvani, A., Coronel, C., Barbosa, A. G., Vega, C., \& Menezes, I. R. (2015). Analysis of bioactivities and chemical composition of Ziziphus joazeiro Mart. using HPLC-DAD. Food chemistry, 186(s/n), 185-191.

Chen, B. M., Liao, H. X., Chen, W. B., Wei, H. J., \& Peng, S. L. (2017). Role of allelopathy in plant invasion and control of invasive plants. Allelopathy J, 41(s/n), 155-166.

Costa, R. G., Medeiros, A. N., Alves, A. R. \& Medeiros, G. R. (2009). Perspectivas de utilização da flor-de-seda (Calotropis procera) na produção animal. Revista Caatinga, 22(1), 276-285.

Fabricante, J. R. Plantas exóticas e exóticas invasoras da Caatinga. Bookess, 2013

Fabricante, J. R.., Oliveira, M. N. A., \& Siqueira Filho, J. A. Aspectos da ecologia de Calotropis procera (Apocynaceae) em uma área de Caatinga alterada pelas obras do Projeto de Integração do Rio São Francisco em Mauriti, CE. (2016). Rodriguésia, 64(3) 647-654.

Ferguson, J. J., Rathinasabapathi, B., \& Chase, C. A. (2013). Allelopathy: How plants suppress other plants. EDIS, 2013(3).

Ferreira, A. G., \& Aquila, M. E. A. (2000). Alelopatia: uma área emergente da ecofisiologia. Revista Brasileira de Fisiologia Vegetal, 12(1), $175-204$.

Gaba, S., Perronne, R., Fried, G., Gardarin, A., Bretagnolle, F., Biju-Duval, L., \& Reboud, X. (2017). Response and effect traits of arable weeds in agro-ecosystems: a review of current knowledge. Weed Research, 57(3), 123-147.

Hierro, J. L., \& Callaway, R. M. (2021). The ecological importance of allelopathy. Annual Review of Ecology, Evolution, and Systematics, 52.

Hoagland, R. E., \& Williams, R. D. (2004). Bioassays-useful tolls of the study of allelopathy. In: Macias, F. A. Galindo, J. C. G., Molinillo, J. M. G., \& Cutler, H. G. (Eds.). Allelopathy: Chemistry and mode of action of allelochemicals. (pp. 315- 341). Boca Raton, Florida: CRC Press. 
Larcher, W. A. (2004). Ecofisiologia vegetal. Rima Artes e Textos.

Leandro, C. S., Bezerra, J. W. A., Rodrigues, M. D. Pereira., S. A K. Fernandes., Silva, D. L; Santos, M. A. F., \& Silva, M. A. P. (2019). Phenolic Composition and Allelopathy of Libidibia ferrea Mart. ex Tul. in Weeds. Journal of Agricultural Science, 11(2), 109-120.

Li, Z. H., Wang, Q., Ruan, X., Pan, C. D., \& Jiang, D. A. (2010). Phenolics and plant allelopathy. Molecules, 15(12), 8933-8952.

Macias, F. A., Gallindo, J. C. G., \& Molinillo, J. M. G. (2000). Plant biocommunicators: application of allelopathic studies. In: Luijendijk, J.C. (Ed.). Years of natural products research - past, present and future. (pp. 137-161). Leiden: Phytoconsult.

Maguire, J. D. Speed of germination-aid in selection and evaluation for seedling emergence and vigor. (1962). Crop Science, Madison, 2(1), $176-177$.

Melo, M. D. S. F., Rocha, C. Q., Santos, M. H., Chavasco, J. M., \& Chavasco, J. K. (2012). Pesquisa de bioativos com atividade antimicrobiana nos extratos hidroetanólicos do fruto, folha e casca de caule do Zizyphus joazeiro Mart. Revista da Universidade Vale do Rio Verde, 10(2), 43-51.

Mendonça, R. L. (2008). Determinação de aleloquímicos por HPLC/UV-VIS em extratos aquosos de sementes de Canavalia ensiformis e estudo da atividade alelopática (Dissertação de Mestrado em Ciências - Química Analítica). Universidade de São Paulo, São Carlos, SP, Brasil.

Oliveira, A. K. D., Coelho, M. D. F. B., Maia, S. S. S., Diógenes, F. É. P., \& Medeiros Filho, S. (2012). Allelopathic activity of different parts of juazeiro (Ziziphus joazeiro Mart.-Rhamnaceae). Acta Botanica Brasilica, 26(3), 685-690.

Oliveira, P. M.L., Dantas, A. M., dos Santos Morais, A. R., Gibbert, L., Krüger, C. C. H., dos Santos Lima, M., \& Borges, G. D. S. C. (2020). Juá fruit (Ziziphus joazeiro) from Caatinga: A source of dietary fiber and bioaccessible flavanols. Food Research International, $129,108745$.

Ortiz, T. A., Gomes, G. R., Takahashi, L. U. S. A., Urbano, M. R., \& Strapasson, E. (2014). Water and salt stress in germinating seeds of pitaya genotypes (Hylocereus spp.). African Journal of Agricultural Research, 9(50), 3610-3619.

Parente, K., Parente Filho, E. G., \& Silva, É. V. (2015). Alelopatia de Ziziphus joazeiro Mart. sobre Lactuca sativa L. e Lycopersicon esculentum Mill.

Pires, N. M., \& Oliveira, V. R. (2001). Alelopatia. In: Oliveira, R. S., \& Constantin, J. (Ed.). Plantas daninhas e seu manejo. (pp. 145-185). Guaíba: Agropecuária.

Prati, D., \& Bossdorf, O. (2004). Allelopathic inhibition of germination by Alliaria petiolata (Brassicaceae). American journal of Botany, 91(2), 285-288.

Popinigis, F. (1985). Fisiologia da semente. (2a ed). Agiplan.

Rezende, A. A., Groppo, M., Ranga, N. T., \& Teixeira, S. P. (2017). Coleta, Herborização e Identificação de Espécies Vegetais. In: Furtado, N. A. J. C.; Veneziani, R. C. S.; Ambrósio, S. R. Coleção Farmácia - Volume 7 - Farmacognosia. Edition: 1, Chapter: 7, Publisher: Atheneu. $103-116$.

Rice, E. L. (1984). Allelopathy (2a ed.). Academyc Press.

Santos, S., \& Rezende, M. O. O. (2008). Avaliação do potencial herbicida de compostos secundários na germinação de sementes de plantas daninhas encontradas em pastagens. Revista Analytica, 32(s/n), 72-78.

Silva, D. L., Santos, M. A. F., Bezerra, J. W. A., Leandro, C. S., Rodrigues, M. D. P., Silva, A. K. F., \& Silva, M. A. P. (2018). Cordia oncocalyx (Allemão) Baill. (Boraginaceae) Chemical Composition and Alelopathic Effect Against Weeds. Journal of Agricultural Science, $10(12), 262-271$.

Silva, R. F., Pacheco, L. P., Soares, L. S., Fonseca, W. L.; Oliveira, J. B. S.; \& Santos, A. S. (2015). Growth suppression of sandspur grass by cover crops. Pesquisa Agropecuária Tropical, 45(3), 319-325.

Silva, J. R. B. D., \& Santos, A. F. D. (2012). Efeito alelopático de extratos aquosos de Senna obtusifolia (L.) H. Irwin e Barneby. Floresta e Ambiente, 17(2), 90-97.

Silva, T. C. L. \& Mendonça, R. L. (2009). Avaliação comparativa de cascas e folhas de Ziziphus joazeiro Mart. (Ramnaceae) em relação aos perfis fitoquímico e toxicológico e as atividades antioxidante e antimicrobiana (Dissertação de Mestrado - Programa de graduação em ciências farmacêuticas). Universidade federal de Pernambuco, Recife, PE, Brasil.

Silva, V. B., Almeida-Bezerra, J. W., de Brito, E. S., Ribeiro, P. R. V., Cordeiro, L. S., Júnior, J. T. C., \& da Silva, M. A. P. (2021). Effect of decomposition of leaves of Azadirachta indica A. Juss. on germination and growth of Myracrodruon urundeuva Allemão. South African Journal of Botany, $142,42-52$.

Souza Neto, M. A. (2016). Ziziphus joazeiro Martius: estudo fitoquímico do extrato hidroetanólico das folhas, fracionamento bioguiado anti-Candida e avaliação do efeito protetor em modelo de doença inflamatória intestinal. (Dissertação - Programa de pós-graduação em ciências framacêuticas). Universidade Federal do Rio Grande do Norte, Natal, RN, Brasil.

Turk, M. A., \& Tawaha, A. M. (2002). Inhibitory effects of aqueous extracts of black mustard on germination and growth of lentil. Pakistan Journal of Agronomy, 1, 1, 28-30.

Villela, F. A., Doni Filho, L., \& Sequeira, E. L. (1991). Tabela de potencial osmótico em função da concentração de polietilenoglicol 6.000 e da temperatura. Pesquisa Agropecuária Brasileira, 26(s/n), 1957-1968.

Zhang, Z., Liu, Y., Yuan, L., Weber, E., \& van Kleunen, M. (2021). Effect of allelopathy on plant performance: a meta-analysis. Ecology Letters, 24(2), 348362 . 